\title{
Plasma FIB Spin Milling Accelerates Battery Research
}

\author{
Bartlomiej Winiarski ${ }^{1,2}$
}

${ }^{1}$ Thermo Fisher Scientific, Brno, Jihomoravsky kraj, Czech Republic, ${ }^{2}$ The Henry Royce Institute, School of Materials, The University of Manchester, Manchester, United Kingdom

*Corresponding author: Bartlomiej.Winiarski@thermofisher.com

The technology for energy storage systems is changing rapidly. In the past decade, the rapid growth of consumer electronics and electric vehicles market leads Li-ion batteries to attract significant attention. Currently two important industries, the energy and automobile industries, are involved in intensive research in this area where the most research takes place into: (a) solid state batteries [1], (b) lithium-ion batteries [2] and (c) sodium-ion batteries [3]. As the energy revolution gathers pace, batteries will be needed for energy storage in order to equalize the fluctuating power production of photovoltaic solar systems or wind turbines. The trend for electromobility places a lot of pressure to accelerate battery research.

Further advancement of their performance for higher energy and better safety is achieved by fundamental understanding of battery materials structures and chemistry throughout the life cycle. Various complex studies have been carries out in situ, ex situ and using 3D correlative multi-scale tomography and microscopy (CMT) [4]. Although CMT is very powerful methodology to gather 3D insight into material microstructure and chemistry $[5,6]$, it requires considerable amount of effort if compared to characterization of materials in two dimensions without prior screening with X-ray micro tomography $(\mu \mathrm{CT})$. In many situations 2D information (SEM, EBSD, EDS, Raman, SIMS) from a cross-section of material give plethora of quantitative and statistical data. Plasma FIB-SEM opened fast access to 100's of microns wide and deep cross-sections allowing collecting multi-modal information with nanometer resolution [6]. Depending on the size of the cross-section, material and type of analyses needed the timeto-results (TTR) can vary from few dozens to hundreds of minutes. Considerable part of TTR is the site preparation time (SPT) for the cross-section: (a) MultiChem gas injection system deposition of a protective layer; (b) milling of the side trenches; (c) milling of the front trench to define of the crosssection and (d) polishing of the cross-section. For example, the SPT time for high resolution electron diffraction pattern analyses (HR-EBSD) over large areas ( $>100$ 's of $\mu \mathrm{m}^{2}$ ) is about $<25 \%$ of the TTR, while the SPT for high resolution SEM imaging is about $>95 \%$ of the TTR. The SPTs for other quantitative techniques sits in between the above percentage values of the TTR. In the recent years TTR was reduced by introducing new more sensitive detection systems, e.g. EDS, EBSD. Further, the femtosecond Laser PFIB-SEM significantly reduced a cross-section preparation or serial sectioning time [7]. Currently, a new large area $\left(<=1 \mathrm{~mm}^{2}\right)$ or large volume acquisition technique, so called Plasma FIB spin milling (PFIB-SM), was introduced by Winiarski at al. [8].

This technique collects data from superficial layers of a specimen surface and does requires minimum or no site preparation time. This work presents for the first time the Plasma FIB spin milling method for applications in the battery research. As an example, a generic NMC cathode from a Li-ion battery cell was spin mill polished, where areas of $500 \mu \mathrm{m}$ in diameter ware prepare within dozens of minutes. The PFIBSM allows collecting curtain-free data from on-axis and off-axis location, thus accessing areas of about $50 \mathrm{~mm}^{2}$. This technique is well suited for rapid surface polishing and collecting multimodal information, while reaching an inert gas transfer condition is possible. 

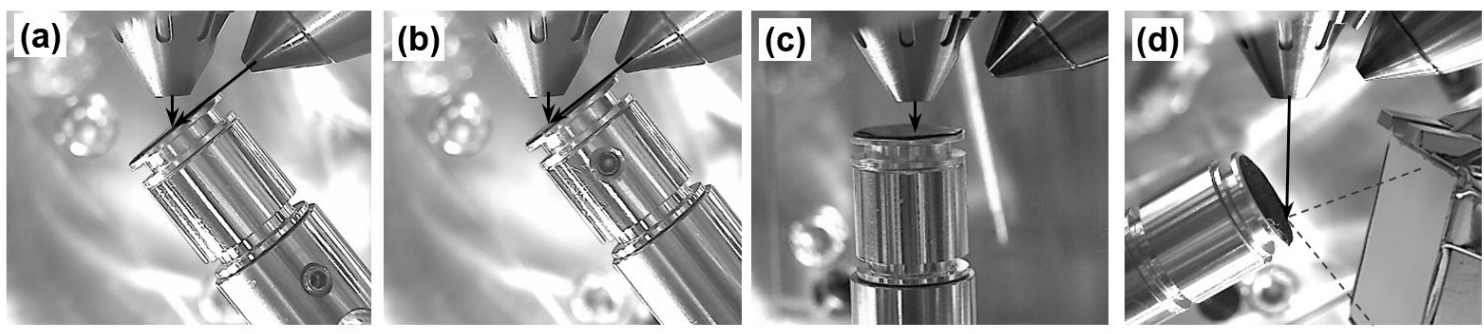

Figure 1. Shows the experimental setup. Hydra PFIB spin milling (a) on axis and (b) off-axis; (c) SEM/EDS data collection and (d) EBSD data collection geometries.
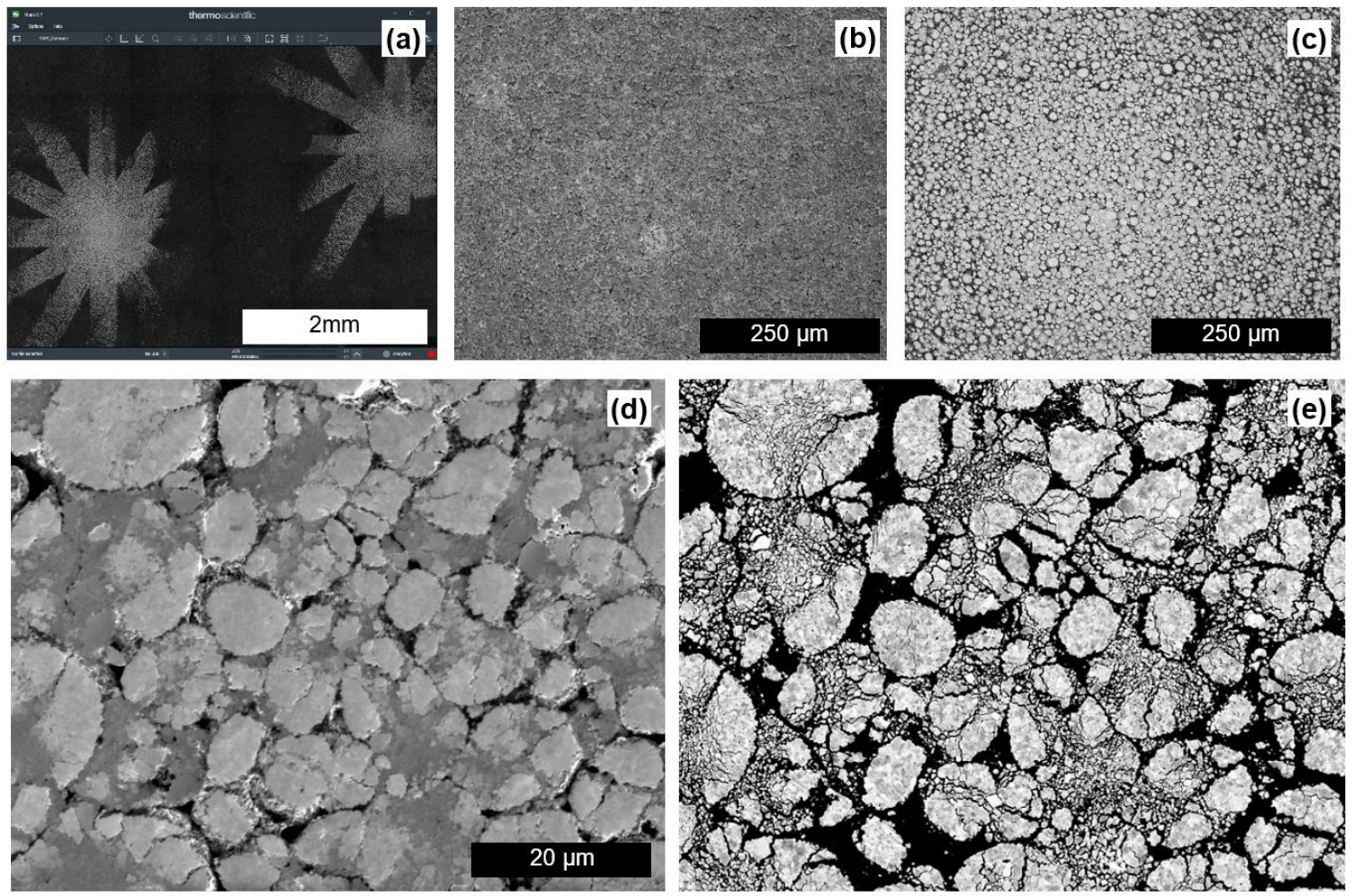

Figure 2. Shows Xe PFIB spin milling results. (a) data is collected in a Maps project; the cathode before (b) and after spin milling (c) @ 30 kV, 60 nA, 1-deg glancing angle, < 60 min; (d) SEM ETD-SE 2kV, $0.8 \mathrm{nA}$; (e) SEM CBS $5 \mathrm{kV}, 1.6 \mathrm{nA}$

\section{References}

[1] J Nanda, C Wang, et al. MRS Bulletin 43(10):740-745.

[2] G Blomgren. Journal of The Electrochemical Society 164(1):A5019-A5025.

[3] M Liu, P Zhang at al. Nature Communications, (2019) 10:3917.

[4] R Moroni, M Börner at al. Scientific Reports 2016; 6: 30109.

[5] B Winiarski, G Pyka, at al. Microscopy and Microanalysis, Nano Supp, Dec 2017.

[6] B Winiarski, G Pyka, at al. Microscopy and Microanalysis 24(S1) 2018:366-367.

[7] SJ Randolph et al., Journal of Vacuum Science \& Technology B36 (2018), p. 06JB01.

[8] B Winiarski, C Rue, at al. Microscopy and Microanalysis 25(S2) 2019:350-351. 